\title{
Autonomous Construction of a Roofed Structure Synthesizing Planning and Stigmergy on a Mobile Robot
}

\section{Conference Paper}

Author(s):

Wismer, Stefan; Hitz, Gregory; Bonani, Michael; Gribovskiy, Alexey; Magnenat, Stéphane

Publication date:

2012

Permanent link:

https://doi.org/10.3929/ethz-a-010007940

Rights / license:

In Copyright - Non-Commercial Use Permitted

Originally published in:

https://doi.org/10.1109/IROS.2012.6386278 


\title{
Autonomous Construction of a Roofed Structure: Synthesizing Planning and Stigmergy on a Mobile Robot
}

\author{
Stefan Wismer ${ }^{1}$, Gregory Hitz ${ }^{1}$, Michael Bonani ${ }^{2}$, Alexey Gribovskiy ${ }^{3}$, and Stéphane Magnenat ${ }^{1,4}$
}

\begin{abstract}
We demonstrate a scenario in which a mobile robot, according to a plan, builds a structure that it can then enter. The robot interacts with the construction using local sensing. This synthesis of planning and stigmergy opens the way to new construction techniques using mobile robots.
\end{abstract}

\section{INTRODUCTION}

Autonomous mobile robots have the potential of supporting construction workers in tedious and dangerous tasks. Current work in this field can be classified into several approaches. A first one is inspired by the actions of eusocial insects like ants and aims at building approximate structures in a scalable way through stigmergy [1], [2]. This method requires only simple robots and a small set of local rules, but the resulting structures are approximate. The second approach focuses on multi-robot algorithms, but still exploits local rules and interactions [3]. The last approach focuses on constructing precise structures according to pre-defined plans, with few sensing of the environment and limited autonomy [4], [5]. Our work aims at a synthesis of these approaches.

This video submission shows a scenario in which a mobile robot builds a roofed structure autonomously. Because the robot must manipulate elements of different sizes into various positions, this setup exhibits many of the challenges a truly autonomous construction robot shall face.

\section{SySTEM DESCRIPTION}

\section{A. Experimental setup}

This experiment aims at assembling a roofed structure (Fig. 1, top). This structure is composed of polystyrene blocks equipped with small magnets to bind them together. The blocks are of three sizes, each a multiple of a basic unit of $6 \times 6 \times 6 \mathrm{~cm}$. The walls consist of layers of small (size 1) and medium (size 2) blocks, while the roof consists of large blocks (size 6). The walls have a height of 3 layers and are 4 units long. At the beginning of the experiment, the 18 blocks are available as 4 lines at pre-defined locations. A VICON tracking system informs the robot of its absolute position.

\section{B. Platform}

Our construction robot is based on the modular marXbot platform [6]. This miniature platform $(17 \mathrm{~cm}$ in diameter) consists of a base, some intermediate layers, and a top

\footnotetext{
1 Autonomous Systems Lab, ETH Zürich, 8092 Zürich, Switzerland.

2 Association Mobsya, 1004 Lausanne, Switzerland

3 LSRO, EPFL, 1015 Lausanne, Switzerland

${ }^{4}$ Corresponding author, email: stephane at magnenat dot net This work was supported by the ASCENS EU FP7-FET and H2SWARM Swiss SNF projects under contract numbers 257414 and 20BI21_134317.
}
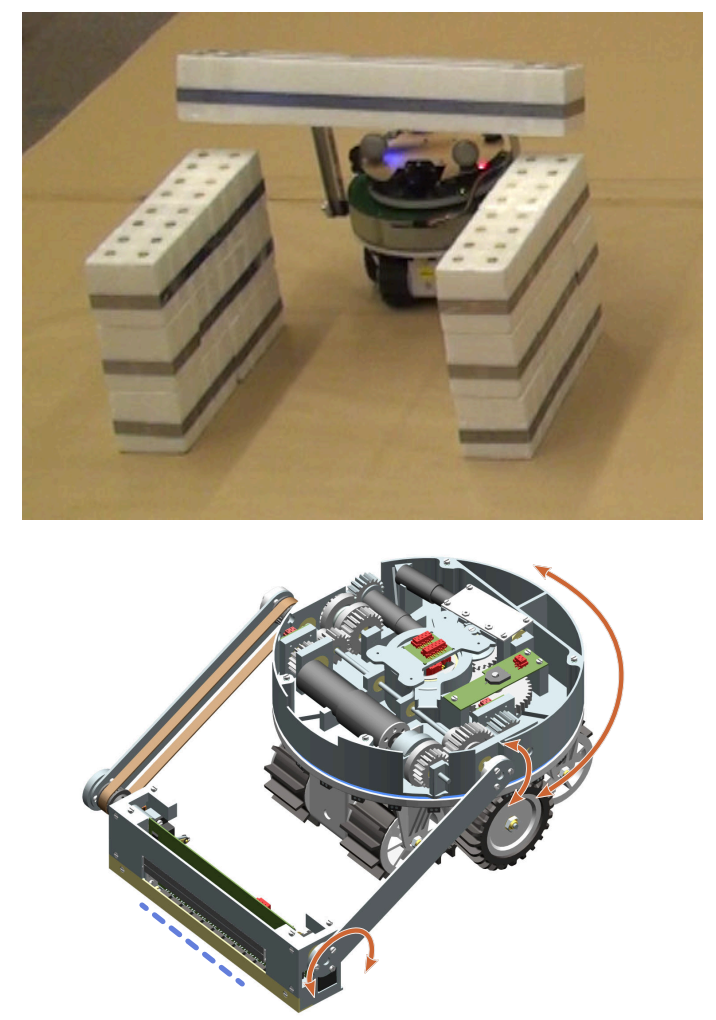

Fig. 1. The experimental setup (top) and the gripper module (bottom).

module. The base provides mobility and energy, and shortrange sensing via a ring of infrared distance sensors. The top module is a Linux-based computer and provides a camera and a Wi-Fi link. Between these modules, we have added a custom-built magnetic-gripper module.

One or more microcontrollers (MCU) drive each module and communicate through a CAN bus. They use ASEBA, an event-based control architecture for microcontrollers [7]. Our gripper module has two micrcocontrollers: one in the body of the robot and the other on the gripper.

\section{Gripper Module}

The magnetic gripper features three degrees of freedom: yaw, elevation, and tilt (Fig. 1, bottom). The resulting work space is large enough so that the robot can access places that are higher than its height and access blocks on its sides.

All axes are compliant and equipped with force sensors. Additionally, two arrays of infrared sensors in the gripper measure the distance to the construction blocks. The gripper can grasp these blocks using a magnetic switchable device [8]. 


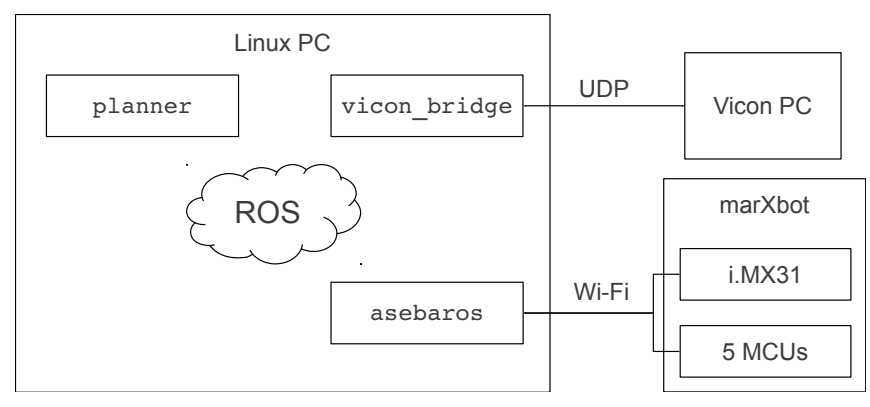

Fig. 2. The software architecture.

\section{Software Architecture}

The software architecture is divided in two layers. A PC with ROS runs the high-level executive. The latter contains a planner that generates a list of actions, such as go to, fetch block, and depose block. This executive receives the pose of the robot from the VICON computer through UDP and communicates with the robot using ASEBA. It orders the execution of the actions and implements the differential-drive control law that moves the robot to requested locations. The low-level layer runs in the microcontrollers and implements the block-manipulation actions such as fetching and deposing.

\section{E. Low-level Actions}

The fetching action aims at grasping a block in its center. A potential misalignment is measured using the difference between the left and the right infrared sensors in the gripper. For the small block, the robot rotates its gripper around the yaw axis, while for the medium and large block the whole robot rotates and scans laterally.

The robot builds walls layer by layer. The behavior differs between the first block of a layer and all subsequent blocks of the same layer. The first block is adjusted with the left edge of the wall using the gripper's infrared sensors. Each subsequent block is then added to the preceding block's right edge. The robot senses the contact between the blocks by measuring the current consumption of the yaw motor.

To construct the roof, the robot centers itself between the two walls using the ring of infrared sensors of its base. The robot then deposes the first block after a certain distance measured by dead reckoning. Each subsequent block is then added adjoining the previous block. The robot senses the contact between the blocks by measuring the force on the elevation axis.

\section{RESUlTS AND DiscusSiON}

All actions are implemented and work most of the times (Table I). Based on these, our robot successfully constructed a wall and a roof. However, out of 10 trials, it did not manage to build two walls and a roof in a single run. Because we execute the actions sequentially, the overall probability of success depends on the successful execution of every action. Yet, the structure consists of 18 blocks, and every block requires 6 actions (one fetch, one depose and 4 go to), therefore building the whole structures demands 108 actions. Assuming that we

\begin{tabular}{llll}
\multicolumn{4}{c}{ Building } \\
\hline build type & success rate \\
wall: first block of layer & $16 / 18$ & \\
wall: other blocks of layer & $11 / 16$ & \\
roof: first block & $6 / 11$ & \\
roof: other blocks & $8 / 11$ & \\
\hline & & Fetching & \\
\hline block type & success rate & average offset & std. dev. \\
\hline Small & $10 / 10$ & $-3.6 \mathrm{~mm}$ & $1.2 \mathrm{~mm}$ \\
Medium & $10 / 10$ & $0.2 \mathrm{~mm}$ & $9.4 \mathrm{~mm}$ \\
Large & $8 / 10$ & $3.8 \mathrm{~mm}$ & $5.4 \mathrm{~mm}$ \\
\hline
\end{tabular}

TABLE I

SUCCESS RATES OF THE LOW-LEVEL ACTIONS

want a success rate of $10 \%$ for the whole construction, every action must have a success rate of $98 \%$, which is larger than our current rates. Thus, the structure presented in the video was built in multiple single runs.

This analysis shows that for long and complex tasks such as building a structure, error detection and recovery is inevitable. This is a non-trivial problem, because errors are of diverse types: they might be misalignments but also ill-placed blocks or lost blocks. Therefore an error-correction mechanism would lead to complex interactions, such as removing a block and replacing it correctly. Moreover, it might also require to do re-planning. We will approach these questions in future work.

\section{CONCLUSION}

In this experiment, a robot manipulated blocks of different sizes into various positions, to build a structure it could then enter. This synthesis of planning and stigmergy opens the way to new construction techniques using mobile robots.

\section{REFERENCES}

[1] R. L. Stewart and R. A. Russell, "A Distributed Feedback Mechanism to Regulate Wall Construction by a Robotic Swarm," Adaptive Behavior, vol. 14, no. 1, pp. 21-51, 2006.

[2] J. Wawerla, G. Sukhatme, and M. Mataric, "Collective construction with multiple robots," in Proceedings of the IEEE/RSJ International Conference on Intelligent Robots and Systems, vol. 3. IEEE Press, 2002, pp. 2696-2701.

[3] J. Werfel and R. Nagpal, "Extended stigmergy in collective construction," IEEE Intelligent Systems, vol. 21, no. 2, pp. 20-28, 2006.

[4] Gramazio and Kohler, "Structural oscillations," http://www.dfab.arch. ethz.ch/web/d/forschung/142.html, 2007-2008, installation at the 11th Venice Architectural Biennale.

[5] B. Khoshnevis, "Automated construction by contour crafting - related robotics and information technologies," Automation in construction, vol. 13, no. 1, pp. 5-19, 2004.

[6] M. Bonani, V. Longchamp, S. Magnenat, P. Rtornaz, D. Burnier, G. Roulet, F. Vaussard, H. Bleuler, and F. Mondada, "The MarXbot, a Miniature Mobile Robot Opening new Perspectives for the Collectiverobotic Research," in 2010 IEEE/RSJ International Conference on Intelligent Robots and Systems (IROS 2010). IEEE Press, 2010.

[7] S. Magnenat, P. Rtornaz, M. Bonani, V. Longchamp, and F. Mondada, "ASEBA: A Modular Architecture for Event-Based Control of Complex Robots," IEEE/ASME Transactions on Mechatronics, vol. PP, no. 99, pp. 1-9, 2010.

[8] F. Rochat, P. Schoeneich, M. Bonani, S. Magnenat, F. Mondada, H. Bleuler, and H. Christoph, "Design of Magnetic Switchable Device (MSD) and applications in climbing robot," in Emerging Trends in Mobile Robotics, H. Fujimoto, M. O. Tokhi, H. Mochiyama, and G. S. Virk, Eds. World Scientific, 2010, pp. 375-382. 